\title{
COMPLICATIONS OF TRANSFORAMINAL EPIDURAL INJECTIONS
}

\section{To the Editor:}

Increased awareness of the number of humans who suffer from unrelieved pain has led to an increase in the treatment of pain, the number of physicians who specialize in the treatment of pain and the number of interventional pain management procedures done. The best data source documenting that increase in the United States is the CMS data tracking procedures performed on Medicare patients. For example, from 1998 to 2001, the number of lumbar transforaminal epidural steroid injections increased form 45,385 to 125,534 , an increase of over $175 \%$ (1). It seems a reasonable assumption that similar increases have occurred in non-Medicare patients.

With this increase in utilization, there has been an increase in the number of anecdotal and case reports of complications. We cannot tell whether the increase is due solely to utilization or whether changes in technique have led to a higher incidence of complications (2-5). Most of the recent concern has been related to epidural steroid injections, sacral, lumbar, thoracic and cervical. Some of these complications have been catastrophic, including monoplegia, paraplegia, quadriplegia, Brown Sequard syndrome and death.

Because of the legal implications associated with adverse events, there is no inclusive database from which we could derive the incidence of complications.

Not having a database also prevents us from being able to conclusively determine the cause of complications. The greatest concern focuses around epidural steroid injections. Complications have

- Been seen in the cervical, lumbar, thoracic and caudal regions and after attempted cervical facet injections;

- Occurred with or without steroids;

- Occurred with particulate and nonparticulate steroids;

- Occurred with transforaminal, interlaminar and caudal approaches

- Occurred with sedation; and

- Occurred with sharp needles.
- There are no reported cases with blunt needles yet.

Based upon case report data, the cause of complications appears to be intra-arterial injections, intra-neural injections and direct penetration of the spinal cord.

At this point, it is not possible to make any practice recommendations. However, the anecdotal evidence is sufficient to raise areas of concern, in which we should focus further investigation.

\section{Sharp versus Blunt Needles}

In animal studies, one cannot easily pierce the viscera with a blunt needle $(6$, 7). Heavner (8) using 20, 22 and 25 gauge blunt and sharp needles replicated these findings. In addition, Heavner (8) demonstrated that blunt needles do not penetrate the nerve root following laminectomy. The use of blunt rather than sharp needles has been recommended (2). Although the use of blunt tip needles seems prudent, we lack clinical evidence that complications with blunt needles would not occur.

A related risk factor is needle movement when changing syringes. The use of low volume, low memory extension tubing would minimize the risk of needle movement when changing syringes.

\section{What is Injected}

Particulate matter injected intravascularly, as in steroid suspensions, is a possible cause of embolic infarcts. However, the injection of non-particulate solutions, such as lidocaine, has also been associated with infarcts. We need greater clarity as whether the use of steroid suspensions, such as Depo-Medrol, is a risk factor. Pressure infarcts can occur from sustained pressure on nerve roots carrying blood supply to the cord or from injectate trapped in epidural loculations causing pressure on the cord. Similarly, subdural spread in multioperated patients with arachnoiditis and scarring can cause pressure infarcts of the cord, cauda equina and nerve roots.

\section{Particulate versus Non-particulate Cor- ticosteroids}

It seems intuitive that adverse cord sequelae due to intra-arterial injection of particulates are more likely due to embolic infarction. However, there is minimal reviewable histopathology to definitively confirm this hypothesis. It has been proposed that non-particulate steroids, e.g. betamethasone phosphate (not Soluspan) or dexamethasone would avert this risk. Two deficiencies prevent us from making this proposal a recommendation. Firstly, the lack of complications with non-particulate injections is not yet apparent from animal studies or human studies. With a low incidence of complications, tens or hundreds of thousands of cases may need to be performed to prove a lower incidence. Recall that chemical infarction with non-particulate medications other than steroids, such as lidocaine, has occurred. Secondly, non-particulates have yet to demonstrate the desired effectiveness in long-term studies.

\section{Approach}

Cervical transforaminal injections have been described as "potentially dangerous (9)." This statement, while correct, is not limited solely to the cervical transforaminal approach and has caused some practitioners to needlessly abandon the approach. We have not yet determined whether there is a higher incidence of complications with a transforaminal versus interlaminar approach. The evaluation of current data suggests that catheter guided interlaminar sitespecific lateral epidural approach will reduce concerns regarding complications. Nor have we confirmed the efficacy of one approach over the other. The interlaminar approach contains the risks of subdural puncture, spinal cord trauma and, more ominously, the possibility of unrecognized entrapment of injectate with epidural loculations, causing delayed pressure cord infarct. Performing interlaminar injections in the prone rather than lateral position runs the risk of the patient "jumping" into the needle, causing needle 
entry into the cord. We have not clarified the best, safest technique.

\section{Sedation}

An alert patient is potentially able to provide a warning if the procedure is going awry. Some centers make it a practice to deeply sedate all patients. The risks of this practice need to be evaluated to determine if patient safety is being compromised for patient comfort.

In conclusion, we are dedicated to the safest possible techniques to provide quality care to our patients. The incidence of complications from interventional techniques is so low that we are currently unable to measure the incidence. Medication management does not effectively solve these problems. Therefore, it behooves us to consider available evidence and look for the best possible therapeutic options for our patients. This communication suggests specific areas which we should further investigate.

\section{REFERENCES}

1. Manchikanti L, Singh V. Interventional Pain Management: Evolving Issues for 2003. Pain Physician 2003; 6:125-137.

2. Nelson JW. Letter to the Editor. In response to Houten JK, Errico TJ. Paraplegia after lumbosacral nerve block. Spine J 2003; 2:88-89.

3. Baker R, Dreyfus P, Mercer $S$ et al. Cervical transforaminal injection of corticosteroids into a radicular artery: A possible mechanism for spinal cord injury. Pain 2003; 103:211-215.

4. Houten JK, Errico TJ. Paraplegia after lumbosacral nerve root block: Report of three cases. Spine I 2002; 2:70-75.

5. Brouwers PJ, Kottink EJ, Simon MA et al. A cervical anterior spinal artery syndrome after diagnostic blockade of the right C6nerve root. Pain 2001; 91:397-399.

6. Berger JJ, Hawkins IF. Celiac plexus injection: Use of a blunt-tipped needle. Regional Anesthesia 1995; 20 (2S).

7. Akins EW, Hawkins IF Jr., Mladinich $C$ et al. The blunt needle: A new percutaneous access device. AJR Am J Roentgenol 1989; 152:181-182.

8. Heavner JE, Racz GB, Jenigiri B et al. Shgarp vs blunt nedle: A comparative study of penetration of internal structures and bleeding in dogs. Pain Practice 2003. In Press

9. Aprill C, Rogers K. Cervical transforaminal injection of corticosteroids. ISIS Scientific Newsletter 2002; 4:21-32.

\section{Standiford Helm II, MD}

Medical Director

Pacific Coast Pain Management Center P.O. Box 2549

Lake Forest, CA 92690-0549

E-mail: drhelm@pcpmc.com

\section{Joseph F. Jasper, MD}

Medical Director

Advanced Pain Medicine Physicians 1628 South Mildred Street, Suite \#105

Tacoma, WA 98465-1613

E-mail: apmedicine@qwest.net

\section{Gabor B. Racz, MD}

Grover Murray Professor, Professor and Chair Emeritus Director Pain Services, Texas Tech University Health Sciences Center Lubbock, TX 79430-0001, E-mail paula.brashear@ttuhsc.edu 